\title{
Two ways of experimental infection of Ixodes ricinus ticks (Acari: Ixodidae) with spirochetes of Borrelia burgdorferi sensu lato complex
}

\author{
Lenka Fišerová, Kateřina Černá, Helena Horká and Jan Kopecký
}

Institute of Parasitology, Biology Centre of the Academy of Sciences of the Czech Republic and Faculty of Science, University of South Bohemia, Branišovská 31, 37005 České Budějovice, Czech Republic

Key words: Lyme disease spirochete, Borrelia burgdorferi, Borrelia garinii, Borrelia afzelii, tick, Ixodes ricinus

\begin{abstract}
A previously reported procedure for the introduction of Borrelia spirochetes into tick larvae by immersion in a suspension of spirochetes was tested on Ixodes ricinus (L.) ticks and three of the most medically important European Borrelia genomic species, B. burgdorferi sensu stricto, B. garinii and B. afzelii. The procedure was compared with "classical" infection of nymphs by feeding on infected mice. Both methods yielded comparable results (infection rate 44-65\%) with the exception of $B$. afzelii, which produced better results using the immersion method (44\%) compared with feeding on infected mice (16\%). Nymphs infected by the immersion method at the larval stage were able to transmit the infection to naïve mice as shown by serology and PCR detection of spirochetal DNA in organs. The immersion method is faster than feeding on infected mice and provides more reproducible conditions for infection. It can be exploited for studies on both pathogen transmission and Borrelia-vector interactions.
\end{abstract}

In Central Europe, Ixodes ricinus (L.) represents the principal vector of human pathogens such as Borrelia burgdorferi, Anaplasma phagocytophilum and tickborne encephalitis virus (Brouqui et al. 2004, Charrel et al. 2004). In Europe, prevalence rates of B. burgdorferi sensu lato in host-seeking nymphs and adults vary between $2 \%$ and $43 \%$ and $3 \%$ and $58 \%$ respectively (Hubálek and Halouzka 1998). Transovarial transmission of spirochetes is possible, but rare (Zhioua et al. 1994).

According to studies on the main American vector of Borrelia spirochetes, Ixodes scapularis, the larvae ingest spirochetes from infected rodents. This results in a population of spirochetes in the midgut, which is transmitted to the nymphal stage. Upon feeding, spirochetes migrate to the salivary glands via the haemolymph and are transmitted to the new host with the saliva (Piesman et al. 1990, Piesman 1995, de Silva and Fikrig 1995). Transmission of a pathogen by tick feeding cannot be compared with intradermal injection of a similar number of spirochetes. First, a feeding tick delivers spirochetes continuously during the feeding period (Crippa et al. 2002) second, tick saliva affects the host immune system facilitating transmission of the pathogen (Nuttall and Labuda 2004).

In many types of experiments including assessment of vaccines against tick-borne pathogens, salivaactivated transmission of pathogens and pathogen-tick interactions, efficient and reproducible infection of all tick developmental stages is required. The standard system for infecting I. scapularis and I. pacificus ticks by feeding on infected hosts was developed by Piesman (1993). More recently, I. scapularis larvae were shown to be infected by immersion in cultures of Borrelia spirochetes (Policastro and Schwan 2003). We examined both methods using the European tick I. ricinus and three medically important Borrelia genomic species, $B$. burgdorferi sensu stricto, B. afzelii and B. garinii.

\section{MATERIALS AND METHODS}

Mice. SPF female $\mathrm{C} 3 \mathrm{H} / \mathrm{HeN}$ mice 8 to 12 weeks old were purchased from Charles River, Sulzfeld, Germany.

Ticks. Ixodes ricinus ticks from a laboratory colony at the Institute of Parasitology, Biology Centre AS CR, České Budějovice, negative in Borrelia burgdorferi sensu lato PCR screening, were used throughout the experiments.

Bacteria. The CB-43 strain of B. afzelii (Štěpánová-Tresová et al. 1999), the CB-53 strain of B. burgdorferi sensu stricto and the CB-61 strain of B. garinii were isolated from $I$. ricinus ticks in the South Bohemia region and identified using genomic species-specific PCR. The spirochetes were grown in Barbour-Stoenner-Kelly-H (BSK-H) medium (Sigma) supplemented with $6 \%$ rabbit serum at $34^{\circ} \mathrm{C}$. Low-passage spirochetes were used ( 2 to 7 passages).

Infection of ticks by feeding on infected mice. Groups of five mice were each inoculated subcutaneously in the dorsal thoracic midline with $10^{3}$ spirochetes of either B. burgdorferi sensu stricto (strain CB-53), B. afzelii (CB-43) or B. garinii (CB-61) respectively in $0.1 \mathrm{ml}$ of BSK-H medium per mouse. One month post infection, groups of 20 nymphs were placed into retaining cells attached to the back of each mouse. Control nymphs were fed on mock-infected mice. After full engorgement the nymphs were maintained at $25^{\circ} \mathrm{C}$ and a relative humidity $80 \%$ until metamorphosis. Ten adults from each mouse were examined for the presence of spirochetes using PCR.

Address for correspondence: J. Kopecký, Institute of Parasitology, Biology Centre of the Academy of Sciences of the Czech Republic, Branišovská 31, 37005 České Budějovice, Czech Republic. Phone: ++420 387775 468; Fax: ++420 385 310 388; E-mail: jan@paru.cas.cz 
Infection of ticks by immersion in cultures of spirochetes. Groups of circa 40 larvae were placed into microcentrifuge tubes ( $n=3$, one tube for each genomic species) with $0.5 \mathrm{ml}$ of spirochete suspension containing $10^{7}$ cells per ml and incubated at $32^{\circ} \mathrm{C}$ for $2 \mathrm{~h}$. Tubes were gently vortexed every $10 \mathrm{~min}$. The control group of 40 larvae was immersed in plain BSK-H medium. Then, the larvae were washed briefly in $70 \%$ ethanol and twice in distilled water and placed on mice to complete engorgement. After moulting, 20 nymphs were examined for the presence of each spirochete genomic species by PCR.

Detection of $B$. burgdorferi sensu lato DNA by PCR. Detection of spirochetal DNA was performed in ticks after moulting for two reasons. First, engorged blood can interfere with PCR; second, the trans-stadial transmission of spirochetes could be determined. JETquick Tissue DNA Spin Kit (Genomed) was used for DNA isolation. Each tick was homogenized in $30 \mu \mathrm{l}$ of PBS and the supernatant was transferred into a microcentrifuge tube with $200 \mu 1$ of T1 buffer and $25 \mu 1$ of proteinase $\mathrm{K}(20 \mathrm{mg} / \mathrm{ml}$, Genomed). Samples were incubated at $56^{\circ} \mathrm{C}$ overnight. Additional steps were performed according to the manufacturer's instructions. For DNA isolation from mouse organs, samples with mass of 15$20 \mathrm{mg}$ were homogenized in $200 \mu \mathrm{l}$ of T1 buffer with $25 \mu$ of proteinase $\mathrm{K}$; further processing was as for tick homogenates. A segment of the B. burgdorferi sensu lato 16S rRNA gene (669 bp) was amplified using forward primer 5'-ACG CTG GCA GTG CGT CTT AA-3' and reverse primer 5'-CTG ATA TCA ACA GAT TCC ACC C-3' (Liebisch et al. 1998). Primers were produced by Generi-Biotech, Czech Republic. PCR was performed in a reaction volume $20 \mu \mathrm{l}$ containing $2.0 \mu \mathrm{l}$ of processed DNA sample, 2.0 $\mu \mathrm{l}$ of Taq buffer (GeneriBiotech), $1.6 \mu \mathrm{l}$ of $10 \mathrm{mM}$ dNTP each (TopBio), $1.0+1.0 \mu \mathrm{l}$ of $0.01 \mathrm{mM}$ oligonucleotide primers, $0.1 \mu \mathrm{l}(2 \mathrm{U})$ Taq DNA polymerase (Generi-Biotech), $12.3 \mu 1$ of $\mathrm{H}_{2} \mathrm{O}$. PCR was performed in an UNO II thermocycler (Biometra). Each sample (after initial DNA denaturation at $95^{\circ} \mathrm{C}$ for $5 \mathrm{~min}$ ) was amplified for 35 repeated cycles of denaturation at $94^{\circ} \mathrm{C}$ for $30 \mathrm{sec}$, annealing at $57^{\circ} \mathrm{C}$ for $30 \mathrm{sec}$, chain elongation at $72^{\circ} \mathrm{C}$ for 1 min. PCR amplification products were resolved on a $1 \%$ agarose electrophoresis gel and visualised under UV light with SYBR Green II (Sigma).

Ability of infected ticks to transmit the infection to mice. Groups of 20 larvae were placed on naïve $\mathrm{C} 3 \mathrm{H} / \mathrm{HeN}$ mice immediately after immersion in the suspension of spirochetes and left to feed to repletion. Two mice were used for each Borrelia genomic species. Nymphs infected as larvae by immersing in the suspension of $B$. afzelii were placed in groups of five on four naïve $\mathrm{C} 3 \mathrm{H} / \mathrm{HeN}$ mice and left to feed to repletion. Two control mice were exposed to nymphs developed from mock-infected larvae. One month later the mice were examined by serology and PCR detection of spirochetes in the heart and urinary bladder was also performed.

ELISA procedure. Wells of high-binding, F96 Maxisorp immunoplates (Nunc, Roskilde, Denmark) were coated with the sonic extract from $B$. burgdorferi sensu stricto strain $\mathrm{CB}$ $53(100 \mu 1$ per well $)$ overnight at $4^{\circ} \mathrm{C}$. The antigen was diluted in a carbonate buffer ( $\mathrm{pH} 9.6$ ) to a concentration $10 \mu \mathrm{g} / \mathrm{ml}$. After blocking with PBS and $10 \%$ newborn calf serum (NCS) for $2 \mathrm{~h}$ at room temperature, mouse sera diluted 1:200 in PBS were added (50 $\mu 1$ per well) and incubated overnight at $4^{\circ} \mathrm{C}$. Plates were then incubated with peroxidase-conjugated swine anti-mouse IgG (Sevak, Prague, Czech Republic) diluted 1:2000 in PBS for one hour at $37^{\circ} \mathrm{C}$. An enzymatic colour reaction was generated using orthophenylendiamine substrate, stopped after 10 min with $2 \mathrm{M} \mathrm{H}_{2} \mathrm{SO}_{4}$ and measured at $490 \mathrm{~nm}$ with an ELISA spectrophotometer (Multiskan MCC/340, Labsystems Oy, Helsinki, Finland). After each incubation the plate was washed at least three times with $0.05 \%$ Tween 20 in PBS. The cut off value was calculated as double the OD value for control sera from non-infected mice.

\section{RESULTS}

\section{Infection of ticks by feeding on infected mice}

Of 15 mice in groups of five, each infected with one of three genomic species of B. burgdorferi sensu lato, almost all mice were seropositive in ELISA one month post infection. Although all sera were tested with the antigen from $B$. burgdorferi sensu stricto (strain CB53 ), the OD exceeded the cut off value due to antigenic cross-reactivity between all three genomic species used (data not shown). Ixodes ricinus nymphs were placed on each mouse and after full engorgement and moulting, adults were examined for the presence of spirochetes by PCR. Table 1 shows the results with ticks infected with particular genomic species of $B$. burgdorferi sensu lato complex. Ticks feeding on all five mice infected with $B$. burgdorferi sensu stricto became infected with the infectivity rate ranging from $20 \%$ to $100 \%$. Taken together, spirochetal DNA was demonstrated in 32 of 50 ticks, which represents $64 \%$. Infection of ticks feeding on mice infected with $B$. afzelii was less efficient. None of the ticks feeding on mice without Borrelia-specific antibodies became infected. The infectivity rate of ticks that had fed on seropositive mice ranged from $0 \%$ to $30 \%$ with the average $16 \%$ (including ticks feeding on

Table 1. Infection of Ixodes ricinus nymphs by feeding on mice infected with Borrelia burgdorferi sensu stricto (strain CB-53), Borrelia afzelii (CB-43) and Borrelia garinii (CB$61)$.

\begin{tabular}{|c|c|c|c|c|}
\hline & \multirow{2}{*}{$\begin{array}{c}\text { Mouse } \\
\text { No. }\end{array}$} & \multirow{2}{*}{ Seroconversion } & \multicolumn{2}{|c|}{ Infection rate } \\
\hline & & & Infected/Total & $\%$ \\
\hline B. burgdorferi & 1 & + & $10 / 10$ & 100 \\
\hline \multirow[t]{5}{*}{ s.s. } & 2 & + & $7 / 10$ & 70 \\
\hline & 3 & + & $9 / 10$ & 90 \\
\hline & 4 & + & $2 / 10$ & 20 \\
\hline & 5 & + & $4 / 10$ & 40 \\
\hline & & Total & $32 / 50$ & 64 \\
\hline \multirow[t]{6}{*}{ B. afzelii } & 1 & + & $2 / 10$ & 20 \\
\hline & 2 & + & $3 / 10$ & 30 \\
\hline & 3 & - & $0 / 10$ & 0 \\
\hline & 4 & + & $3 / 10$ & 30 \\
\hline & 5 & + & $0 / 10$ & 0 \\
\hline & & Total & $8 / 50$ & 16 \\
\hline \multirow[t]{6}{*}{ B. garinii } & 1 & - & $0 / 10$ & 0 \\
\hline & 2 & + & $8 / 10$ & 80 \\
\hline & 3 & + & $4 / 10$ & 40 \\
\hline & 4 & + & $5 / 10$ & 50 \\
\hline & 5 & + & $5 / 10$ & 50 \\
\hline & & Total & $22 / 50$ & 44 \\
\hline
\end{tabular}


Table 2. Infection rate of Ixodes ricinus nymphs infected at the larval stage by immersion in suspension of three genomic species of Borrelia burgdorferi sensu lato.

\begin{tabular}{|c|c|c|}
\hline \multirow{2}{*}{ Borrelia genomic species } & \multicolumn{2}{|c|}{ Infection rate } \\
\hline & Infected/Total & $\%$ \\
\hline B. burgdorferi s.s. & $13 / 20$ & 65 \\
\hline B. afzelii & $8 / 20$ & 40 \\
\hline B. garinii & $10 / 20$ & 50 \\
\hline Total & $321 / 60$ & 52 \\
\hline
\end{tabular}

Table 3. Infectivity to mice of nymphs infected at the larval stage by immersion in suspension of Borrelia afzelii.

\begin{tabular}{lccc}
\hline Mouse & \multirow{2}{*}{ Seroconversion } & \multicolumn{2}{c}{ Spirochetes in organs* } \\
\cline { 3 - 4 } No. & + & Hearth & Urinary bladder \\
\hline 1 & - & + & + \\
2 & - & - & + \\
3 & + & + & - \\
4 & - & - & + \\
$5^{* *}$ & - & - & - \\
$6^{* *}$ & & - & - \\
\hline
\end{tabular}

*Spirochetal DNA was detected by PCR; **Control mice infested with Borrelia-free nymphs.

the seronegative mouse). Together $44 \%$ of nymphs were infected by feeding on mice infected with $B$. garinii. One mouse was seronegative with $0 \%$ of infected nymphs; the infection rate for the remaining four mice ranged from $40 \%$ to $80 \%$.

Regardless of the Borrelia genomic species, a total of $61 / 150$ nymphs $(41 \%)$ became infected. The highest tick infection rate was achieved with $B$. burgdorferi sensu stricto, the lowest with B. afzelii.

\section{Infection of ticks by immersion in a suspension of Borrelia spirochetes}

Ixodes ricinus larvae were immersed in the suspension of one of three Borrelia genomic species. After moulting to nymphs, they were examined for the presence of spirochetes by PCR. The results are summarized in Table 2 . The most efficient infection $(65 \%$ of infected ticks) was achieved with $B$. burgdorferi sensu stricto (strain CB-53). Borrelia garinii was more infective than B. afzelii ( $50 \%$ versus $40 \%$ of infected ticks).

\section{Ability of infected ticks to transmit infection to the new host}

Mice on which the larvae were placed immediately after immersion in the suspension of spirochetes, were examined for the presence of spirochetal DNA or Borrelia-specific antibodies one month post engorgement. No evidence of transmission of Borrelia spirochetes from the larvae to mice was observed.

To demonstrate the ability of immersion-infected larvae to maintain $B$. afzelii infection trans-stadially and to transmit the spirochetes to the host, moulted nymphs were fed on naïve mice. One month post engorgement specific antibodies were detected in two of four mice. Further examination of tick-exposed mice by PCR showed spirochetal DNA in the heart and urinary blad- der of both seropositive mice and in the urinary bladder of one of seronegative mice. The fourth mouse and both control mice were negative in the PCR assay (Table 3).

\section{DISCUSSION}

The purpose of this study was to examine two methods of experimental infection of Ixodes ricinus ticks with three genomic species from the Borrelia burgdorferi sensu lato complex pathogenic for humans. We exploited a standard method of tick infection by feeding on infected mice (Piesman 1993) and the more recently developed method based on immersion of tick larvae in a suspension of spirochetes (Policastro and Schwan 2003). The first procedure resembles the natural route of infection. Mice can be infected intradermally or subcutaneously or via feeding of infected ticks (Piesman 1993) or by inoculation of spirochetes mixed with tick salivary gland extract (Pechová et al. 2002). Another factor which can influence the success of host-to-tick transmission is the infectious dose used for host infection. While Piesman (1993) obtained a tick infection rate ranging between $18 \%$ and $83 \%$ for three strains of B. burgdorferi sensu stricto, using inoculum of $10^{7}$ spirochetes per host, we achieved a rather high infection rate $(64 \%)$, using mice infected with a much lower dose $\left(10^{3}\right.$ spirochetes per mouse).

The different infection rates obtained for particular Borrelia genomic species cannot be connected with the vector competence of $I$. ricinus tick. In Europe, the most prevalent genomic species is $B$. afzelii, followed by $B$. garinii; $B$. burgdorferi sensu stricto is the least frequent (Rauter and Hartung 2005). The different infection rates obtained in our experiments may relate to the different virulence of the strains used, which can depend on the number of passages in vitro. Various isolates of the same Borrelia genomic species can vary markedly in their infectivity for ticks or hosts (Peavey and Lane 1996) and even one Borrelia strain can represent a heterogeneous mixture of clones varying in morphology, growth rate, protein profile, plasmid content and infectivity (Elias et al. 2002).

Infection rates achieved by immersion of $I$. ricinus larvae in the suspensions of Borrelia spirochetes were similar to infection rates of nymphs fed on infected mice. While $B$. burgdorferi sensu stricto was again the most infectious, the lowest infection rate was observed for B. afzelii. Values varied from $40 \%$ to $65 \%$ depending on the genomic species, compared with $16 \%$ to $64 \%$ for nymphs fed on infected mice. The percentage of nymphs infected with B. burgdorferi sensu stricto (65\%) was identical with the infection rate of $I$. scapularis larvae immersed in a suspension of spirochetes of the same genomic species (Policastro and Schwan 2003). Policastro and Schwan (2003) also demonstrated that immersion-infected larvae were able to transmit the pathogen to the host immediately after immersion. This transmission was not demonstrated in our experiments. 
Mice on which immersion-infected larvae were placed to complete engorgement, did not seroconvert and Borrelia DNA was not detected in their organs by PCR. The difference between our experiments and those by Policastro and Schwan (2003) consists in "holding time", the time for which the larvae were allowed to recover after immersion in the suspension of spirochetes before being applied to mice. While the holding time used by Policastro and Schwan was $18 \mathrm{~h}$ to 3 days, in our design the larvae were placed on mice immediately after immersion. This way the engorgement efficiency could be increased to $50-70 \%$ (data not shown) compared with 10-50\% reported by Policastro and Schwan (2003). On the other hand, spirochetes in larvae placed on mice without a holding time could have not enough time to adapt to the inner tick milieu to be able to pass from the midgut to salivary glands and infect the host.

To show that nymphs moulted from immersioninfected larvae are able to infect the host, nymphs infected with $B$. afzelii (infection rate $40 \%$ ) were placed on $\mathrm{C} 3 \mathrm{H} / \mathrm{HeN}$ mice in groups of five and allowed to feed to full engorgement. Three of four mice became infected as demonstrated by detection of spirochetal DNA in the heart and urinary bladder. One of these infected mice was negative in ELISA, which confirms the limita- tions in serology testing as a method for demonstrating Borrelia infection (Tylewska-Wierzbanowska and Chmielewski 2002).

The present work shows that the procedure of tick infection by immersion in a suspension of Borrelia spirochetes can be successfully exploited for the infection of I. ricinus with the three most important European genomic species of the $B$. burgdorferi sensu lato complex. This method is faster than feeding on infected mice and yields comparable infection rates. Although representing an artificial method of tick infection, like capillary feeding (Fingerle et al. 2002), it provides more reproducible conditions for infection and the following life stages (nymph and adult) can be used for studies on pathogen transmission to naïve and tick- or pathogenimmune hosts as well as for the analysis of Borreliavector interactions.

Acknowledgements. The work was supported by grant 524/05/0811 from the Grant Agency of the Czech Republic. This study is a part of the research project of the Institute of Parasitology, Academy of Sciences of the Czech Republic (Z60220518), Centre for Molecular Ecology of Vectors and Pathogens (LC06009), and of the Faculty of Science, University of South Bohemia, České Budějovice (MSM 600766 5801).

\section{REFERENCES}

Brouqui P., Bacellar F., Baranton G., Birtles R.J., BJoËrSDORFF A., BlanCO J.R., CARUSO G., CINCO M., Fournier P.E., Francavilla E., JenSENIUS M., KAZAR J., Laferl H., Lakos A., Lotric Furlan S., Maurin M., Oteo J.A., Parola P., Perez-Eid C., Peter O., Postic D., Raoult D., Tellez A., Tselentis Y., Wilske B. 2004: Guidelines for the diagnosis of tick-borne bacterial diseases in Europe. Clin. Microbiol. Infect. 10: 1108-1132.

Charrel R.N., Attoui H., Butenko A.M., ClegG J.C., Deubel V., Frolova T.V., Gould E.A., Gritsun T.S., HeINZ F.X., LABUDA M., LASHKEVICH V.A., LOKTEV V., LUNDKVIST A., Lvov D.V., Mandl C.W., Niedrig M., PaPa A., Petrov V.S., Plyusnin A., RANDOlPh S., SUSS J., Zlobin V.I., DE LAMBALLERIE X. 2004: Tick-borne virus diseases of human interest in Europe. Clin. Microbiol. Infect. 10: 1040-1055.

CRIPPA M., RAIS O., GERN L. 2002: Investigations on the mode and dynamics of transmission and infectivity of Borrelia burgdorferi sensu stricto and Borrelia afzelii in Ixodes ricinus ticks. Vector-Borne Zoonotic Dis. 2: 3-9.

DE SILVA A.M., FIKRIG E. 1995: Growth and migration of Borrelia burgdorferi in Ixodes ticks during blood feeding. Am. J. Trop. Med. Hyg. 53: 397-404.

Elias A.F., SChMUTZhard J., STEWART P.E., SChWAN T.G., RosA P. 2002: Population dynamics of a heterogeneous Borrelia burgdorferi B31 strain in an experimental mouse-tick infectious cycle. Wien. Klin. Wochenschr. 114: 557-561.

Fingerle V., Rauser S., Hamer B., Kahl O., Heimerl C., SChUlte-SPeChtel U., GeRn L., WilsKe B. 2002: Dynamics of dissemination and outer surface protein expression of different European Borrelia burgdorferi sensu lato strains in artificially infected Ixodes ricinus nymphs. J. Clin. Microbiol. 40: $1456-1463$.
HubÁleK Z., HAlouZKA J. 1998: Prevalence rates of Borrelia burgdorferi sensu lato in host-seeking Ixodes ricinus ticks in Europe. Parasitol. Res. 84: 167-172.

LIEBISCH G., SOHNS B., BAJTECH W. 1998: Detection and typing of Borrelia burgdorferi sensu lato in Ixodes ricinus ticks attached to human skin by PCR. J. Clin. Microbiol. 36: 33553358.

NUTTALL P.A., LABUDA M. 2004: Tick-host interactions: salivaactivated transmission. Parasitology 129: S177-189.

PEAVEY C.A., LANE R.S. 1996: Comparison of infectivities of six tick-derived isolates of Borrelia burgdorferi for rodents and ticks. J. Clin. Microbiol. 34: 71-75.

PECHOVÁ J., ŠTĚPÁNOVÁ G., KovÁŘ L., KOPECKÝ J. 2002: Tick salivary gland extract-activated transmission of Borrelia afzelii spirochaetes. Folia Parasitol. 49: 153-159.

PIESMAN J. 1993: Standard system for infecting ticks (Acari: Ixodidae) with the Lyme disease spirochete, Borrelia burgdorferi. J. Med. Entomol. 30: 199-203.

PIESMAN J. 1995: Dispersal of the Lyme disease spirochete Borrelia burgdorferi to salivary glands of feeding nymphal Ixodes scapularis (Acari: Ixodidae). J. Med. Entomol. 32: $519-521$.

PIESMAN J., OLIVER J.R., SINSKY J.R. 1990: Growth kinetic of the Lyme disease spirochete (Borrelia burgdorferi) in vector tick (Ixodes dammini). Am. J. Trop. Med. Hyg. 42: 352-357.

POLICASTRO P.F., SCHWAN T.G. 2003: Experimental infection of Ixodes scapularis larvae (Acari: Ixodidae) by immersion in low passage cultures of Borrelia burgdorferi. J. Med. Entomol. 40: 364-370.

RAUTER C., HARTUNG T. 2005: Prevalence of Borrelia burgdorferi sensu lato genospecies in Ixodes ricinus ticks in Europe: a metaanalysis. Appl. Environ. Microbiol. 71: 7203-7216. 
ŠTĚPÁNOVÁ-TRESOVÁ G., KOPECKÝ J., KUTHEJLOVÁ M. 1999: Identification of Borrelia burgdorferi sensu stricto, Borrelia garinii and Borrelia afzelii in Ixodes ricinus ticks from Southern Bohemia using monoclonal antibodies. Zbl. Bakteriol. 289: 797-806.

TYLEWSKA-WIERZBANOWSKA S., CHMIELEWSKI T. 2002: Limitation of serologic testing for Lyme borreliosis: evaluation of

Received 26 October 2007
ELISA and Western blot in comparison with PCR and culture methods. Wien. Klin. Wochenschr. 114: 601-605.

Zhioua E., AESCHLIMANN A., GeRn L. 1994: Infection of fieldcollected Ixodes ricinus (Acari: Ixodidae) larva with Borrelia burgdorferi in Switzerland. J. Med. Entomol. 31: 763-766.

Accepted 7 December 2007 